Ann. Sci. forest., 1979, 36 (4), 299-320.

\title{
Télédétection d'attaques de la Cochenille Matsucoccus feytaudi duc. \\ (Coccoidea, Margarodidae) dans les forêts de pins maritimes du Sud-Est de la France par microdensitométrie trichrome sur film I.R.C.
}

\author{
J. RIOM, Ch. GOILLOT * et J. P. FABRE ** \\ Laboratoire de sylviculture et d'écologie de la pinède landaise, I.N.R.A., \\ Domaine de l'Hermitage, Pierroton, \\ 33610 Cestas-Principal \\ * Laboratoire de Télédétection \\ Centre national de Recherches agronomiques, I.N.R.A., \\ 78000 Versailles \\ ** Station de Zoologie forestière, \\ Centre de Recherches d'Avignon. I.N.R.A., \\ Avenue Vivaldi, Avignon
}

\begin{abstract}
Résumé
Le niveau des populations de cochenilles et les symptômes de l'attaque ont été étudiés pour chaque arbre de plusieurs peuplements de Pins maritimes qui ont servi à la mise au point d'une méthode d'étude par télédétection à partir de prises de vues IRC à basse altitude $(35,75$ et $375 \mathrm{~m})$ et à faible échelle $(1 / 250,1 / 500,1 / 2500)$. Les couronnes ont été étudiées en microdensitométrie trichrome ; la densité des couches vertes $(\mathrm{dv})$ et bleves $(\mathrm{db})$ est significative de l'attaque : les arbres sains ont des valeurs $d v$ et $d b$ élevées $(1,8$ à 2$)$, les arbres très attaqués des valeurs $d v$ et $d b$ faibles $(1,2$ à 1,3$)$, les arbres en cours d'attaque se situent entre ces deux extrêmes. La couche rouge du film (sensible à l'infra-rouge) n'est pas significative des degrés d'attaque. Par cette méthode les arbres peuvent être répartis en six classes.

Le film IRC numérisé en deux cent cinquante-six niveaux en microdensitométrie trichrome ef traité sur une unité interactive à microprocesseurs permet une classification et une quantification automatiques des pixels correspondant aux divers degrés d'attaque du peuplement.
\end{abstract}

En Forêt encore plus qu'en Agriculture les biologistes, sont désarmés lorsqu'ils doivent cartographier sur de grandes surfaces des attaques d'insectes ravageurs, de champignons ou des agressions dues à des polluants; les attaques d'insectes sont souvent fugaces et non prévisibles et une enquête au sol demande la disposition de très gros moyens concentrés sur une courte période.

Pour ces raisons de nombreux chercheurs ont étudié les possibilités d'utilisation de la télédétection en se servant de prises de vues photographiques en noir et blanc panchromatique, en couleur, ou en fausse couleur infrarouge (IRC) ; plus récemment l'utilisation de radiomètres à balayage portés par des avions ou des satellites a ouvert de nouvelles voies. 


\section{1. - Choix du programme}

\section{1. - Le problème}

En 1956 des foyers de dépérissement de Pin maritime ont été signalés dans la région de Bormes-Les-Mimosas et de Saint-Tropez. Ces foyers se sont progressivement étendus et actuellement on peut considérer que les 100000 ha de forêts de Pins maritimes du sud-est de la France ont été touchés avec plus ou moins d'importance selon les stations.

Les mécanismes de ce " dépérissement » sont actuellement bien connus grâce aux travaux de Carle, Fabre, Riom et Schvester. Les dégâts sont provoqués par une cochenille Margarodidae, Matsucoccus feytaudi Duc. Cette dernière signalée pour la première fois en 1940 dans les Landes a été introduite accidentellement dans la région Maures-Estérel, à une date qu'il est difficile de préciser.

Elle a trouvé des conditions favorables à son développement; elle a pullulé, colonisé toute la région Maures-Estérel et détruit la plus grande partie des peuplements de Pin maritime. Les travaux de Riom et al. ont analysé les mécanismes de cette pullulation par une étude comparée de la biologie, de la phénologie et de l'écologie de l'espèce dans l'ensemble de l'aire du Pin maritime (France, Espagne, Italie, Portugal, Maroc) en insistant plus particulièrement sur la comparaison entre les populations de M. feytaudi dans le sud-est et le sud-ouest de la France :

- dans le sud-est la cochenille introduite, a pullulé rapidement, s'est dispersée dans la quasi-totalité des peuplements à partir des foyers initiaux et a détruit les peuplements adultes de Pins maritimes;

- dans le sud-ouest l'espèce est très largement répandue ; on peut même estimer, sans évidemmení pouvoir le vérifier, que la totalité des peuplements adultes hébergent du M. feytaudi.

Le niveau des populations de la cochenille est dans les Landes à un niveau relativement bas à cause, en grande partie, des conditions microclimatiques du printemps. La coïncidence entre la période d'éclosion des larves de la cochenille et la présence d'eau dans les fissures des écorces est le principal facteur de mortalité de M. feytaudi. De la coïncidence plus ou moins parfaite de ces périodes dépendent les variations du taux de multiplication annuel de l'espèce ; il n'est pas exclu qu'une série d'années favorables à la cochenille puisse se présenter provoquant ainsi une pullulation et des dégâts dans les forêts du sud-ouest de la France. Une fois le processus de dégradation enclenché les coléoptères xylophages peuvent prendre le relais et de ravageurs secondaires passer au rang de ravageurs principaux contribuant ainsi à la destruction de la forêt, même si entre temps la pullulation de $M$. feytaudi a subi une régulation par les facteurs climatiques du printemps, redevenus défavorables.

\section{2. - But des opérations}

La production forestière du sud-ouest de la France est capitale pour l'économie régionale et nationale. Si une pullulation de $M$. feyfaudi se produisait dans ce massif forestier qui couvre plus d'un million d'hectares, il serait primordial de connaître l'intensité et le nombre des foyers avant de mettre en œuvre les moyens d'intervention. 
Une enquête au sol, même si elle bénéficie de la collaboration des organismes forestiers ne pourra «cartographier» sur une telle surface les points de pullulation d'où la nécessité d'une réflexion sur un programme de télédétection. Ce programme devrait déterminer la possibilité d'utilisation, aussi bien des photographies, que des images obtenues à l'aide de scanners aéroportés ou placés à bord de satellites pour :

- dans un premier temps, détecter des foyers de dépérissement provoqués par M. feytaudi ;

- dans un second temps, contrôler l'efficacité des moyens de lutte employés.

N'ayant pas constaté de pullulation de $M$. feytaudi dans la région landaise, il a été décidé d'effectuer les opérations de mise au point méthodologique dans une zone de pullulation du sud-est. On peut en effet admettre que des résultats obłenus dans le sudest seront transposables à une autre région à la condition d'effectuer, si nécessaire, un «calage » prenant en compte les varialions de la réponse des arbres sains d'une région à l'autre.

De plus cette méthodologie, éiant donné la finesse recherchée, doit être transposable à l'étude d'autres problèmes entomologiques ou pathologiques, au moins pour les forêts de Pins.

\section{2. - Observations biologiques au sol}

\section{1. - Choix des stations d'étude}

Il a été déterminé en fonction des critères suivants :

- Age du peuplement.

15 à 20 ans correspond à la tranche d'âge idéal, car les estimations de populations de $M$. feytaudi et la notation des symptômes au sol sont plus simples que dans des peuplements plus âgés. La surface des houppiers est suffisamment importante pour permettre une bonne discrimination des arbres à une altitude de 300 à $400 \mathrm{~m}$.

- Structure du peuplement.

La répartition des arbres doit être la plus régulière possible ; elle ne peut jamais être parfaite car les peuplements de Pin maritime du sud-est sont pratiquement toujours issus de régénérations naturelles, très denses et non dépressés.

- Végétation arbustive et herbacée.

Elle doit être réduite, l'idéal étant son absence totale afin d'éliminer les effets "parasites» dus à la végélation dans le cas où un dépouillement automatique est utilisé.

- Situation de la parcelle.

Pour limiter les effets de perspective la parcelle idéale doit être horizontale et la plus plate possible ; étant donné le relief très accidenté de la région Maures-Estérel elle doit être presque obligatoirement en fond de vallée. 
- Niveau de dépérissement dans le peuplement.

Le peuplement doit être en début de phase de mortalité ce qui permet d'avoir tous les intermédiaires entre les arbres sains et les arbres morts.

Plusieurs parcelles dans lesquelles la dynamique des populations de $M$. feytaudi était suivie depuis quelques années et qui correspondaient aux critères de choix ont été retenues (fig. 1) :

- Saint-Cassien,

- La Mole,

- Les Campaux,

- Bagnols en Forêt.

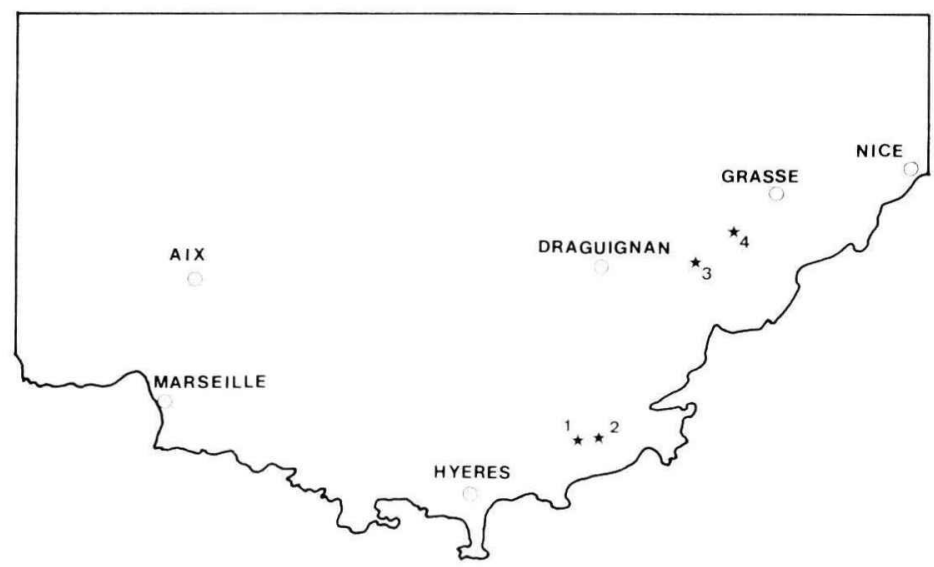

FIG. 1. - Situation des stations étudiées par télédétection dans la région Maures-Estérel

(1. Les Campaux ; 2. La Môle ; 3. Bagnols en Forêt ; 4. Saint Cassien).

Location of sites investigated by remote sensing in the Maures-Esterel area

(1. Les Campaux ; 2. La Môle ; 3. Bagnols en Forêt ; 4. Saint Cassien).

Dans la parcelle Bagnols en Forêt les études biologiques ont été nombreuses ; elles ont été beaucoup plus limitées dans les trois autres.

\section{2. - Caractéristiques de la station «Bagnols en forêt»}

La parcelle correspond à une ancienne vigne colonisée depuis une vingtaine d'années par Pinus pinaster, Calluna vulgaris, Cistus albidus, Cistus monspeliensis... ; elle est sur rhyolite permienne et a un pH de 4 à 5 .

D'une surface de 1 à 1,5 ha elle est limitée de tous côtés par des chemins non goudronnés assurant un bon repérage topographique en cas d'utilisation de scanners (fig. 2).

Le peuplement est en majorité dans une classe d'âge de 15 à 20 ans avec en bordure quelques individus dominants de 40 à 50 ans.

La densité relativement importante de Pins maritimes a empêché la colonisation par la Callune et les Cistes qui restent localisés sur les bordures. Le sol est pratiquement nu dans la plus grande partie de la parcelle. 


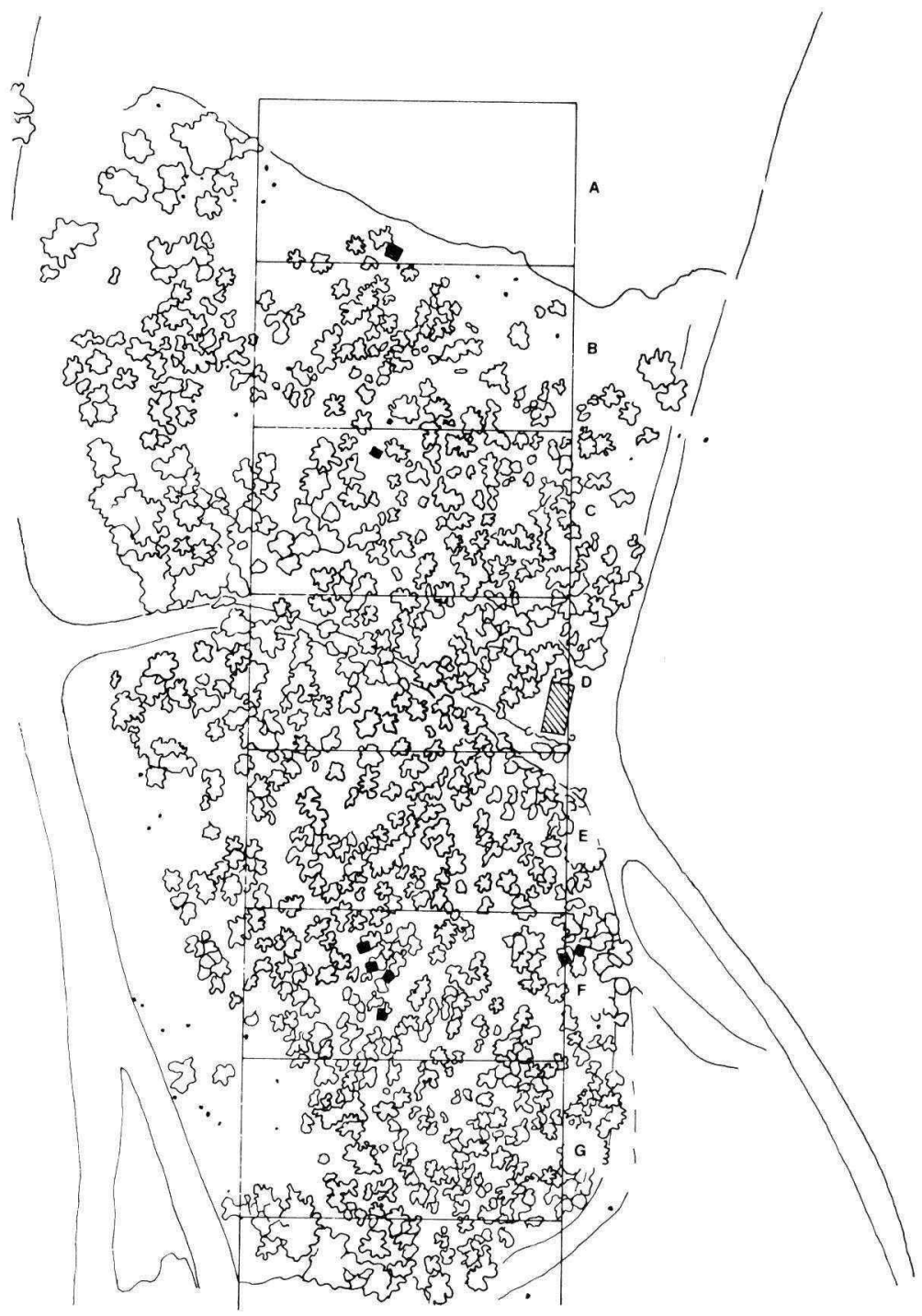

FIG. 2. - Découpage du peuplement Bagnols.

Subdivision of the Bagnols stand.

Trois abris météorologiques classiques (équipés de thermohygrographes enregistreurs), un pluviomètre enregistreur, deux abris météo ventilés (équipés de sondes humides et sèches) informent en permanence sur le microclimat du peuplement. Un abri météo témoin installé sur un sol dégagé à quelques centaines de mètres du peuplement fournit des indications sur le mésoclimat.

La parcelle est attaquée depuis plusieurs années par la cochenille mais le taux de mortalité des arbres est encore relativement faible ; il a contribué à éclaircir légèrement le peuplement et les quelques trouées causées par cette attaque n'ont pas encore été colonisées par la végétation spontanée. 


\section{3. - Observations entomologiques au sol}

Dans la station étudiée par télédétection le niveau d'infestation était suivi depuis plusieurs années pour les besoins d'une analyse de la dynamique des populations de M. feytaudi.

\subsubsection{Etude des populations de la cochenille.}

Certains arbres (une cinquantaine) traités chimiquement, donc indemnes de population naturelle de la cochenille ont servi de témoin.

Trois cents arbres distribués dans le peuplement ont été analysés individuellement grâce à une méthode de piégeage adhésif des mâles (Riom, 1977) ; cette méthode d'une précision relative donne une bonne idée de la population présente sur chaque arbre, surtout si elle est uitilisée plusieurs années consécutives sur les mêmes arbres. En effet d'un arbre à l'autre, selon son âge, sa forme, son épaisseur d'écorce, elle capture un taux variable de la population réelle, mais d'une année sur

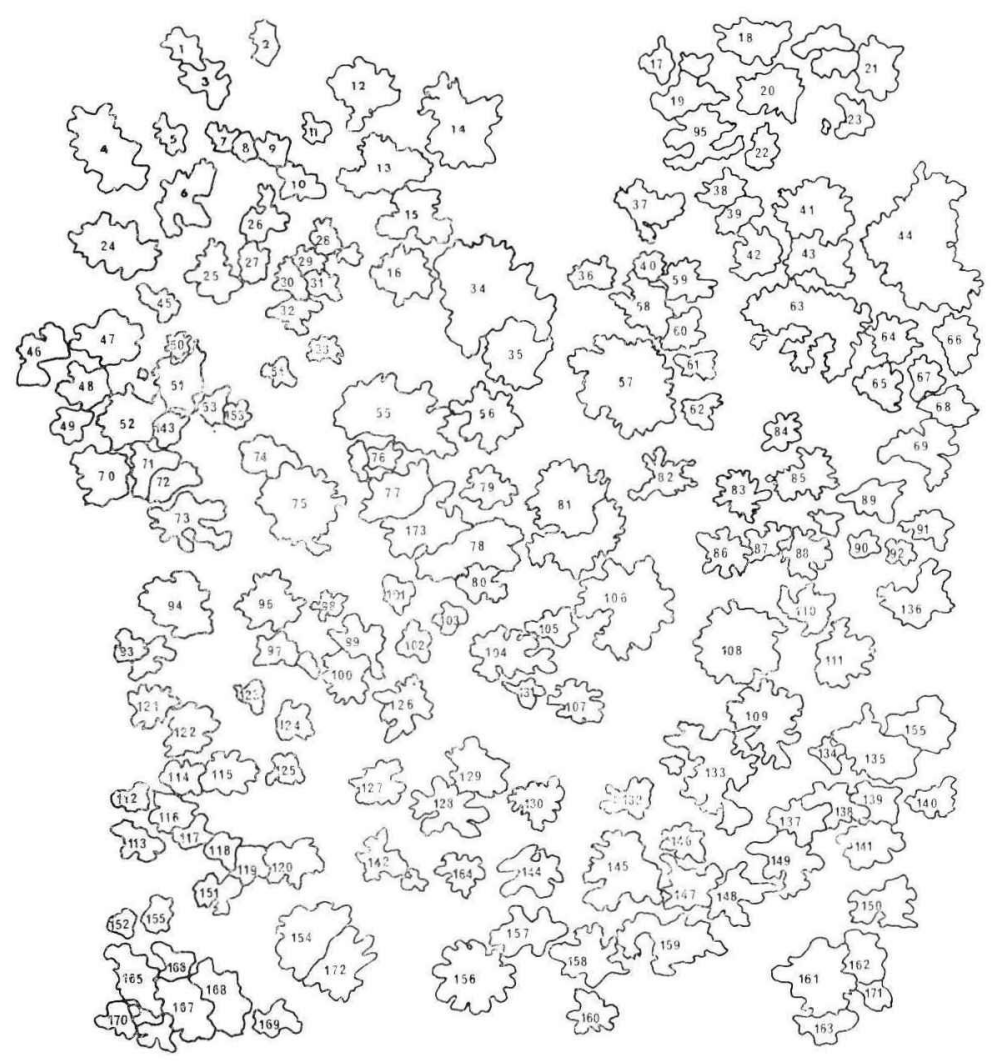

FIG. 3. - Type de plan utilisé pour l'étude du niveau des populations dans le peuplement Bagnols et le repérage des arbres étudiés en microdensitométrie (vue partielle de la figure 2).

Type of map used in the investigation of populations in the Bagnols stand and the locating of trees studied by means of microdensitometry (partial view of figure 2). 
l'autre et pour le même arbre, elle capture un taux presque identique en registrant ainsi les variations réelles des populations.

Il est important de noter qu'étant donné le type de répartition de Matsucoccus dans le peuplement, les arbres doivent être étudiés individuellement : un arbre peu infesté voisine parfois avec un arbre mort depuis quelques années, indiquant par là une colonisation progressive du peuplement par la cochenille. II s'écoule 6 à 8 ans entre la première infestation d'un arbre et sa mort.

2.32. Etude des symptômes de l'attaque.

L'analyse des populations de la cochenille complétée par l'observation individuelle des arbres et leur notation en fonction de leur état de dépérissement. Ces observations simples, effectuées deux fois par an, (printemps et automne) tenaient compte des catégories suivantes :

1. aucun symptôme,

2. quelques symptômes (jaunissement d'extrémités),

3. symptômes bien visibles,

4. symptômes très visibles,

5. arbre presque mort,

6. arbre mort.

L'augmentation des populations de $M$. feytaudi Duc. est très progressive sur l'arbre, puisqu'il faut 5 à 7 ans pour qu'un arbre soit colonisé. L'insecte, comme toutes les cochenilles, enfonce ses stylets dans les tissus vivants, suce la sève et injecte dans l'arbre des phytotoxémiases : tout ceci conduit à une progressivité des symptômes sur plusieurs années, quoiqu'il y aił une extériorisation plus imporiante, chaque année, au printemps et à l'automne.

Dans la classification précédente les catégories 3 et 4 sont souvent regroupées car elles tiennent trop compte de l'interprétation du notateur. Ces notations ont un double but :

- examiner si d'autres ravageurs que M. feyiaudi n'interfèrent pas dans les symptômes, et par là même éliminer les arbres «douteux» ;

- étudier la valeur des méthodes de télédétection, ef leur possibilité de détecter une attaque avant qu'elle ne soit visible au sol, même pour un observateur averti (déteciion précoce).

Ce second point est important, mais considéré comme insuffisant pour rejeter définitivement une méthode de télédélection car le premier objectif était d'étudier les possibilités d'analyses rapides ef sur de grandes surfaces des dégâis provoqués par M. feytaudi Duc.

\section{4. - Cartographie des peuplements}

Elle a été établie en utilisant les clichés fournis par des vols au $1 / 250^{\circ}$ et au $1 / 500^{\circ}$; chaque arbre est caractérisé sur la carle par le contour de son houppier, les clichés étant en recouvrement stéréoscopique de 60 p. 100, seule la partie centrale de chacun a été utilisée afin de corriger au maximum les effets de perspectives. Pour les bordures parallèles à l'axe de vol, les houppiers ont été positionnés sur la base du fût, en général 
très visible ef la géométrie corrigée approximativement. Pour certains secteurs dans lesquels les houppiers étaient serrés il a été indispensable de compléter la cartographie par une vérification au sol. Cette cartographie exacte est indispensable pour éviter les erreurs au niveau des notations de dégâts et des estimations de populations de Matsucoccus.

Sur la parcelle de Bagnols-en-Forêt plus de mille deux cents arbres ont ainsi été individualisés et numérotés, chaque individu pouvant être retrouvé sans erreur, soit sur le terrain, soit sur les clichés (fig. 3).

\section{3. - Opérations en vol}

Les quatre parcelles indiquées figure 1 étaient inscrites dans le programme «Ressources terrestres » du Centre National d'Etudes Spatiales; les vols de février et juin 1971 ainsi que celui de novembre 1972 ont été pris en charge par le C.N.E.S. Le vol du 8 septembre 1971 a été financé par l'I.N.R.A. grâce à une aide de la D.G.R.S.T. Le tableau 1 résume les opérations effectuées sur les stations. La présente note ne concerne que la mission du 8 septembre 1971 dont les documents à grande échelle ont servi à l'étude des possibilités de l'émulsion IRC pour la caractérisation des attaques parasitaires sur les forêts de résineux.

\section{1. - Caractéristiques du vol}

Le vol est exécuté avec un avion monomoteur (Aéromacchi, type AL 60) de l'O.F.P. $\left({ }^{1}\right)$, équipé avec un matériel de prise de vue adapté aux vols à très basse altitude, comprenant :

- une caméra Omera 33, équipée d'un objectif kinoptic de $150 \mathrm{~mm}$, avec compensation de filage, asservissement de diaphragme, réglage du tirage. Il est possible d'introduire, dans la caméra, les paramètres de vol de façon à obtenir un optimum de netteté des clichés. La faible profondeur de champ de l'optique aux hauteurs de vol les plus basses $(37 \mathrm{~m})$ ne permet pas d'obtenir une égale netteté au niveau du sol et à la hauteur des couronnes des arbres lorsque ceux-ci dépassent 2 à $3 \mathrm{~m}$;

- un dispositif de calcul de vitesse sol et de mesure de dérive ;

- un radio altimètre pour la mesure des hauteurs de vol au-dessus du sol.

La correction de tirage affichée dans la caméra est calée d'après l'écho radar du sol. La rétrodiffusion par les houppiers intervenant peu dans ces problèmes, il s'ensuit une certaine défocalisation des images et, partant, quelques défauts de netteté pour les houppiers des arbres de plus de 2 à $3 \mathrm{~m}$ de hauteur. Ce léger manque de netteté n'est pas gênant pour l'étude microdensitométrique des houppiers.

Le film utilisé a une largeur de $127 \mathrm{~mm}$ (5 inches) ; l'image utile est au format $115 \times 115 \mathrm{~mm}$.

(1) Omnium Français de photogramétrie 06 Nice. 


\section{TABLEAU 1}

Missions effectuées sur les sites d'étude depuis 1971

Aerial surveys over the experimental sites since 1971

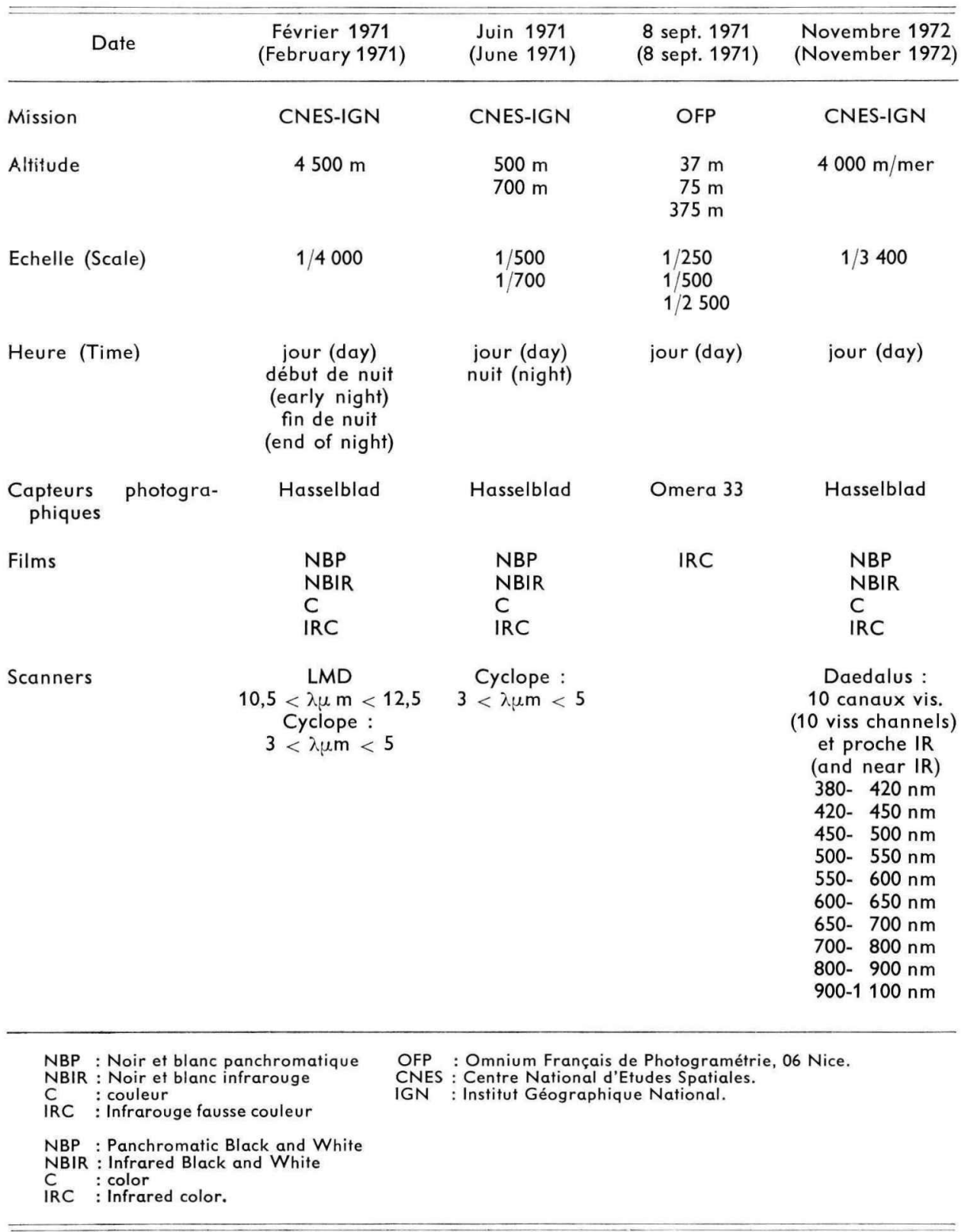




\section{2. - Emulsion et filtrage}

Le film IRC (infra-rouge couleur) Aerochrome Infrared (Kodak type 2443) est dérivé d'une émulsion mise au point durant la dernière guerre mondiale et destinée à séparer la végétation verte des objets peints en vert (Tarkington, R. G., 1963).

L'utilisation de ce film est très délicate. De nombreux échecs sont dus à un mauvais temps d'exposition, qui doit être réglé très précisément, au vieillissement rapide de l'émulsion $\left(^{1}\right)$ et au filtrage mal adapté au problème à étudier. C'est ainsi qu'un certain nombre d'expérimentateurs tels Benson et Sims (1967) Ciesla, Bell et Curlin (1967) ont pu affirmer que ce type d'émulsion ne présente pas d'intérêt pour l'étude des pro-

\section{TABLEAU 2}

Caractéristiques microdensitométriques de 23 arbres de la mission du 8 septembre 1971 Microdensitometric characteristics of 23 trees from survey 8 september 1971

\begin{tabular}{|c|c|c|c|c|c|c|c|c|c|c|c|c|}
\hline \multirow{2}{*}{$\begin{array}{l}\text { Parcelle } \\
\text { (Lot) }\end{array}$} & \multirow{2}{*}{$\begin{array}{l}\text { Réf. } \\
\text { (Réf.) }\end{array}$} & \multirow{2}{*}{$\begin{array}{l}\text { Arbre } \\
\text { (Tree) }\end{array}$} & \multicolumn{3}{|c|}{$\begin{array}{l}\text { Support (Optical } \\
\text { density of film) }\end{array}$} & \multicolumn{3}{|c|}{$d \max$} & \multicolumn{3}{|c|}{$\begin{array}{l}\text { Couronnes } \\
\text { (Crowns) }\end{array}$} & \multirow{2}{*}{$d v \times d b$} \\
\hline & & & $d v$ & $\mathrm{db}$ & $d r$ & $d v$ & $d b$ & $d r$ & $d v$ & $d b$ & $d r$ & \\
\hline Campeaux & $\begin{array}{l}\mathrm{Rd} 1 \\
\mathrm{Rd} 1 \\
\mathrm{Rd} 1 \\
\mathrm{Rd} 2\end{array}$ & $\begin{array}{l}A \\
B \\
C \\
A\end{array}$ & 0,31 & 0,40 & 0,34 & 2,62 & 2,29 & 3,39 & $\begin{array}{l}1,60 \\
1,08 \\
1,50 \\
1,51\end{array}$ & $\begin{array}{l}1,46 \\
1,55 \\
1,26 \\
1,41\end{array}$ & $\begin{array}{l}0,67 \\
0,65 \\
0,55 \\
0,74\end{array}$ & $\begin{array}{l}2,34 \\
1,67 \\
1,89 \\
2,13\end{array}$ \\
\hline La Mole & $\begin{array}{l}\text { Rd } 3 \\
\text { Rd } 3 \\
\operatorname{Rd} 4 \\
\operatorname{Rd} 5 \\
\operatorname{Rd} 5\end{array}$ & $\begin{array}{l}\text { A } \\
B \\
A \\
A \\
B\end{array}$ & $\begin{array}{l}0,28 \\
0,25 \\
0,29\end{array}$ & $\begin{array}{l}0,32 \\
0,34 \\
0,38\end{array}$ & $\begin{array}{l}0,29 \\
0,39 \\
0,42\end{array}$ & $\begin{array}{l}2,64 \\
2,71 \\
2,67\end{array}$ & $\begin{array}{l}2,26 \\
2,15 \\
2,28\end{array}$ & $\begin{array}{l}3,32 \\
3,47 \\
3,43\end{array}$ & $\begin{array}{l}1,30 \\
1,16 \\
1,89 \\
1,63 \\
1,67\end{array}$ & $\begin{array}{l}1,08 \\
1,24 \\
1,67 \\
1,46 \\
1,46\end{array}$ & $\begin{array}{l}0,57 \\
0,54 \\
0,68 \\
0,68 \\
0,76\end{array}$ & $\begin{array}{l}1,40 \\
1,44 \\
3,16 \\
2,38 \\
2,44\end{array}$ \\
\hline Bagnols & $\begin{array}{l}\text { Rd } 6 \\
\operatorname{Rd} 6 \\
\operatorname{Rd} 6 \\
\operatorname{Rd} 6 \\
\operatorname{Rd} 7 \\
\operatorname{Rd} 7 \\
\operatorname{Rd} 8 \\
\operatorname{Rd} 8 \\
\operatorname{Rd} 8 \\
\operatorname{Rd} 8 \\
\operatorname{Rd} 9 \\
\operatorname{Rd} 10 \\
\operatorname{Rd} 10 \\
\operatorname{Rd} 10\end{array}$ & $\begin{array}{c}\mathrm{n}^{\circ} 7 \\
16 \\
97 \\
108 \\
86 \\
91 \\
94 \\
103 \\
115 \\
123 \\
90 \\
\mathrm{~A} \\
\mathrm{~B} \\
\mathrm{C}\end{array}$ & $\begin{array}{l}0,29 \\
0,29\end{array}$ & $\begin{array}{l}0,39 \\
0,39\end{array}$ & $\begin{array}{l}0,34 \\
0,36\end{array}$ & $\begin{array}{l}2,68 \\
2,71\end{array}$ & $\begin{array}{l}2,30 \\
2,37\end{array}$ & $\begin{array}{l}3,37 \\
3,51\end{array}$ & $\begin{array}{l}1,61 \\
1,42 \\
1,40 \\
1,51 \\
1,54 \\
1,45 \\
1,75 \\
1,78 \\
1,20 \\
1,44 \\
1,60 \\
1,28 \\
1,22 \\
1,40\end{array}$ & $\begin{array}{l}1,42 \\
1,24 \\
1,19 \\
1,44 \\
1,33 \\
1,27 \\
1,50 \\
1,69 \\
1,19 \\
1,43 \\
1,55 \\
1,19 \\
1,25 \\
1,37\end{array}$ & $\begin{array}{l}0,61 \\
0,56 \\
0,54 \\
0,61 \\
0,62 \\
0,58 \\
0,76 \\
0,71 \\
0,59 \\
0,69 \\
0,80 \\
0,65 \\
0,67 \\
0,76\end{array}$ & $\begin{array}{l}2,29 \\
1,76 \\
1,67 \\
2,17 \\
2,05 \\
1,84 \\
2,63 \\
3,01 \\
1,43 \\
2,06 \\
2,48 \\
1,52 \\
1,53 \\
1,92\end{array}$ \\
\hline \multicolumn{3}{|c|}{ Moyenne (Mean) } & 0,28 & 0,37 & 0,36 & 2,66 & 2,26 & 3,41 & & & & \\
\hline
\end{tabular}

Légende : dv : densité couche magenta

$\left.\begin{array}{l}\mathrm{db}: \text { densité couche jaune } \\ \mathrm{dr}: \text { densité couche cyan }\end{array}\right\}$ en développement positif

dv : optical density of magenta layer

$\left.\begin{array}{l}\mathrm{db}: \text { optical density yellow layer } \\ \mathrm{dr} \text { : optical density cyan layer }\end{array}\right\}$ positive development 
blèmes phytosanitaires. Cependant des travaux beaucoup plus nombreux tels que ceux de : Heller R. C. et Bean J. L., 1952 ; Meyer M. P. et French D. W., 1967 ; Murtha P. A. et Hamilton L. S., 1969 ; Murtha P. A., 1976 entre autre montrent un intérêt.

En usage normal on utilise avac ce type de film un filtre ja une $n^{0} 15$ qui coupe le spectre en dessous de $520 \mathrm{~nm}$, ou un ître no 12 qui coupe à $500 \mathrm{~nm}$, supprimant ainsi une partie des bleus. Avec ce filtre les feuillus apparaissent en rouge vif et les résineux en bleu plus ou moins violacé. Cette dernière teinte est souvent saturée, rendant ainsi une interprétaíion densitométrique difficile. Ces différences entre feuillus et résineux s'expliquent par le fait qu'au-dessus de $750 \mathrm{~nm}$ la réflectance des feuillus est supérieure à 50 p. 100 alors que celle des résineux est plus faible, (Rodriguez-Bejarano, 1975). De ce fait, les résineux sont non seulement sous-exposés dans le visible à cause de leur teinte sombre, donc beaucoup plus foncés sur la diapositive finale, mais également leur faible réflectance dans le proche infra-rouge conduit à avoir un film sur la couche cyan (sensible au proche infra-rouge) peu exposée donc très foncée sur le positif. En réalité le phénomène est plus complexe car les diverses couches du film ne sont pas totalement indépendantes.

Dans cette écude on a filtré en coupant en dessous de $540 \mathrm{~nm}$ (filtre équivalent à un Wratten 21) ; ce filtrage élimine la dominante bleue ef les résineux apparaissent en rouge foncé, ce qui est inhabituel avec les fiims IRC. II n'est plus possible alors de les distinguer des feuillus qui apparaissent avec les mêmes tonalités de rouge, ce qui n'est pas grave dans le cas présent.

\section{3. - Développement du film}

L'émulsion Kodak aerochrome infra-rouge 2443 est développée suivant les techniques de développement en vigueur en Europe. Pour cette émulsion les techniques

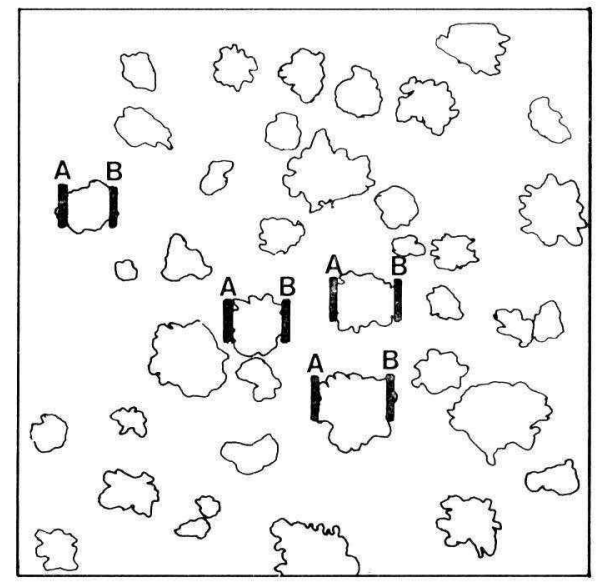

FIG. 4. - Contour des cimes au rapport 1/1 sur un cliché IRC pris à basse altifude.

La densitométrie est faite entre les repères $A$ et $B$.

Outlines of tree crowns (1/1) on a low altitude IRC photograph.

Densitometric analysis was performed between guide marks A and B.

(1) L'émulsion IRC doit être conservée à très basse fempérature $\left(-75{ }^{\circ} \mathrm{C}\right.$ dans les laboratoires spécialisés ef aumoins à $-20^{\circ} \mathrm{C}$ ) à la température ambiante ses caractéristiques sont (en partie) altérées au bout de 48 h environ. 
permettent de se rapprocher des caractéristiques de balance des couleurs définies par Kodak Rochester sans, toutefois, atteindre celles-ci parfaitement. II est donc illusoire de rechercher des corrélations absolues entre les phénomènes et les couleurs enregistrées.

La figure 4 montre l'un des clichés étudié par microdentométrie sur lequel les houppiers sont repérés.

\section{4. - Etude microdensitométrique}

Pour un domaine spectral donné une densitométrie globale peut être effectuée sur un cliché par procédé optique (détermination de l'opacité ou inversement du coefficient de transmission), ou bien par un procédé électronique (balayage de l'image par un pinceau lumineux, codage des niveaux du signal recueilli par un détecteur et transcription des données sur un support informatique ou sur un téléviseur couleur) ou encore par des procédés purement photographiques.

Dans cette étude nous avons utilisé la microdensitométrie trichrome qui seule permet l'étude fine de la réponse de l'émulsion, et assure une bonne reproductibilité de l'analyse d'un vol à l'autre.

\section{1. - Technique utilisée}

\section{- Microdensitométrie}

La microdensitométrie des houppiers repérés sur les clichés est effectuée suivant la méthode déjà citée par Rossetti (Rossetti et al., 1966 ; Rossetti, 1967). Les mesures sont faites avec un microdensitomètre Joyce Lœbel, type MK 3 CS, $n^{\circ} 1180$, à double faisceau, dont les caractéristiques sont résumées dans le tableau 3.

\section{TABLEAU 3}

Caractéristiques du microdensitomètre Joyce Lœbel type MK 3 CS et des filtres utilisés

Characteristics of the Joyce Lobel microdensitometer - type MK 3 CS and of the filters used

- objectif : $\times 5$

- Sensibilité : $0,13 \mathrm{~d} / \mathrm{cm}$

- Coin optique : F 819

- Echelle en X: $\times 10$

- filtres : status A de la Sté MTO bleu DA $439 \mathrm{C}$ vert DA $532 \mathrm{C}$ rouge DA $640 \mathrm{C}$
- Objective lens : $\times 5$

- Sensitiveness : $0,13 \mathrm{~d} / \mathrm{cm}$

- Optical wedge : F 819

- Scale in X: X10

- Filters : A Status, MTO Soc. blue DA $439 \mathrm{C}$ green DA $532 \mathrm{C}$ red DA $640 \mathrm{C}$

Des mesures de calage sont effectuées préalablement pour corriger les données fournies par le microdensitomètre :

- densité optique nulle : valeur de la densité optique obtenue en l'absence de document ; 
— densité optique du support du film (ESTAR) ; cette donnée est obtenue après suppression de l'émulsion ; sitif).

- densité optique maximale prise dans la zone non exposée (opaque en diapo-

La figure 5 met en correspondance les différentes caractéristiques du film IRC Kodak 2443 avec celles des couleurs des trois couches (les pics des courbes de transmission des couches jaunes, magenta et cyan se situent respectivement à 444, 549 et $666 \mathrm{~nm}$ ) qu'il comporte et la transmission du jeu de filtres Status A.

1. Caracteristiques du film IRC Kodak 2443

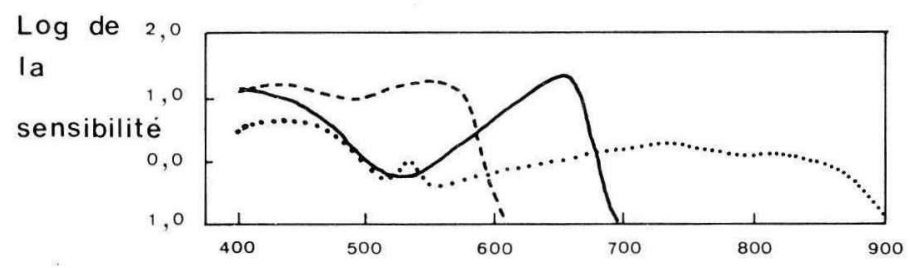

2.Caracteristiques des colorants

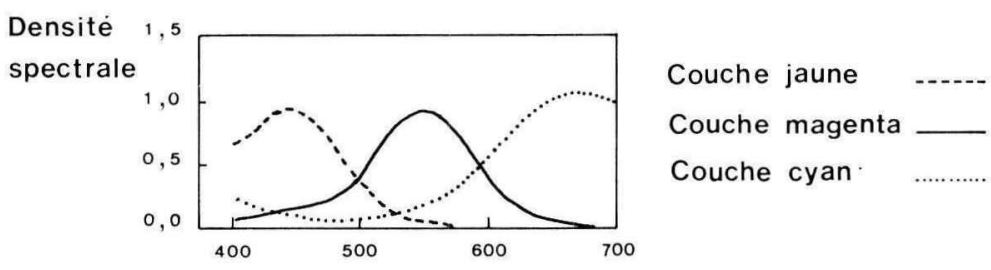

3. Caracteristiques des filtres

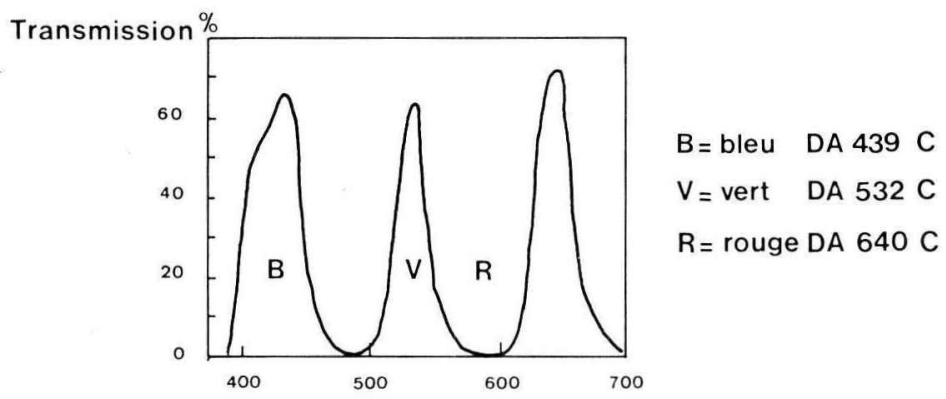

FIG. 5. - Caractéristiques du film et des filtres utilisés dans cette étude.

Characteristics of films and filters used.

La microdensitométrie est effectuée en procédant à un balayage du houppier repéré entre les repères $A$ et $B$ (fig. 4). On choisit empiriquement une largeur de fente qui permet d'obtenir un maximum d'intégration compatible avec la surface de la plage photographique représentant la courronne. La fente qui a été choisie avait une 
hauteur de $1 \mathrm{~mm}$ (perpendiculairement au sens du balayage) et une largeur de 0,4 $\mathrm{mm}$. Ce balayage est effectué successivement dans les trois bandes spectrales fournies par les trois filtres du jeu Status A (tabl. 3).

Après correction des niveaux de référence, on calcule la moyenne arithmétique du profil obtenu pour chaque houppier.

ref $\operatorname{Rd} 8.94$

arbre sain

ref $\operatorname{Rd} 8.123$

arbre en cours

d'attaque

ref $\operatorname{Rd} 8.115$

arbre très

attaque
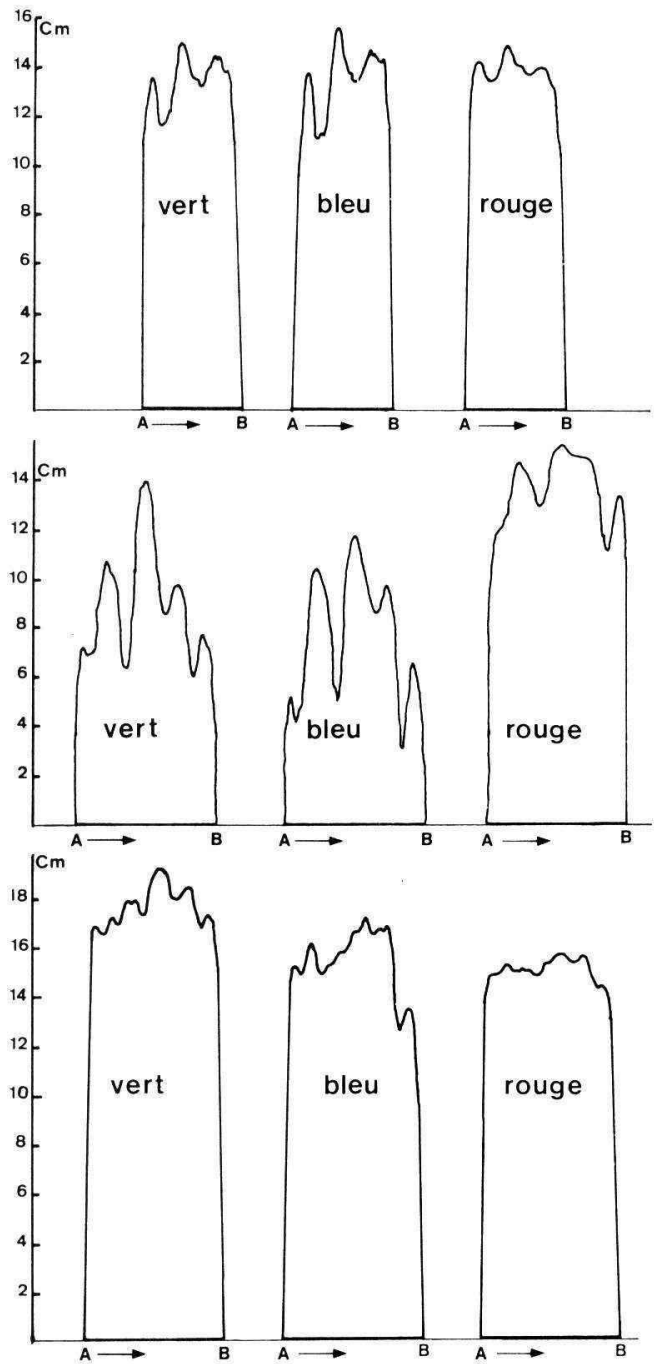

FIG. 6. - Densitogrammes obtenus pour un arbre sain (Rd 8-94), un arbre en cours d'attaque (Rd 8-123) et un arbre fortement atfaqué (Rd 8-115). La mesure est effectuée avec les caractéristiques suivantes : bras $1 / 10$, fente $0,4 \times 1 \mathrm{~mm}$, densité : $0,130 / \mathrm{cm}$; les valeurs exactes de densité et les corrections sont indiquées sur le tableau 2.

Densitograms obtained for a healthy tree (Rd 8-94), a tree undergoing a attack (Rd 8-123) and a heavily attacked tree (Rd 8-115). Measurements were based on the following characteristics : ratio arm $1 / 10$; slit $0,4 \times 1 \mathrm{~mm}$; optical density $0,130 / \mathrm{cm}$. Exact optical density values and rectifications are given in table 2. 
Les densités prises sur le support sans émulsion ainsi que sur les parties non exposées ( $d$ max) permettent d'estimer le taux maximum d'erreur cumulées. Pour chacune des trois couches il ne dépasse pas $\pm 0,10 \mathrm{~d}$ et, généralement il est voisin de la moitié de cette valeur.

\section{2. - Résultats}

La figure 6 donne un exemple de trois densitométries sur la couronne de 3 arbres entre les repères $A$ et $B$.

Sur les couronnes, l'amplitude de variation la plus faible s'observe pour la couche cyan (= couche IR) : minimum 0,54 d, maximum 0,76 d.

Les variations les plus fortes se notent pour la couche magenta (sensible au rouge) minimum 1,08, maximum 1,89. Ces variations sont donc bien supérieures au taux maximum d'erreur estimé et donc significatives.

Pour la couche sensible au vert, les densités varient de 1,08 minimum à 1,69 maximum. C'est donc apparemment dans les deux domaines spectraux auxquels ces couches sont sensibles (couche jaune, compte tenu du filtrage de 540 à $600 \mathrm{~nm}$, couche magenta de 540 à $710 \mathrm{~nm}$ environ) qu'il faut rechercher les variations de réponse densitométrique liées aux variations de réflectance des couronnes.

Nous ne nous préoccuperons plus, dans ce qui suit des réponses dans le proche infra-rouge, puisqu'elles ne sont pas significativement différentes, pour les différentes couronnes.

Les résultats obtenus s'expliquent d'après le mécanisme qui régit les réponses densitométriques de l'émulsion tricouche inversible 2443 , en fonction de l'énergie spectrale émise.

Les réponses respectives de chacune des trois couches de l'émulsion ne sont pas spectralement précises, et il y a superposition nettement dans le vert et le rouge, ce qui ajouté à la répartition du spectre solaire permet d'expliquer le résultat obtenu.

\section{3. - Interprétation}

L'ensemble des résultats obtenus est regroupé dans le tableau 2.

On remarquera que la couche rouge est la moins affectée des trois et que la discrimination entre les différents arbres s'effectue à partir des couches verte et bleue préférentiellement.

Le graphique en figure 7 met en évidence la répartition des degrés d'attaques en fonction des variations de densité optique $d v$ et $d b$ dans les couches verte et bleue respectivement. II montre aussi que les valeurs $d v$ et $d b$ sont corrélées et que l'étude densitométrique d'une seule couche suffit pour classer les arbres selon leur degré d'attaque. Par sécurité, cependant, il est préférable de tenir compte d'un indice de vigueur que l'on peut caractériser par le produit $d v \times d b$.

On obtient ainsi le classement suivant :

$$
\mathrm{dv} \times \mathrm{db}>3,2 \text { : arbres sains ou très peu infestés ; }
$$

$3,2>d v \times d b>2,7$ : arbres en cours d'attaque ;

$d v \times d b<2,7$ : arbres très dépérissants. 


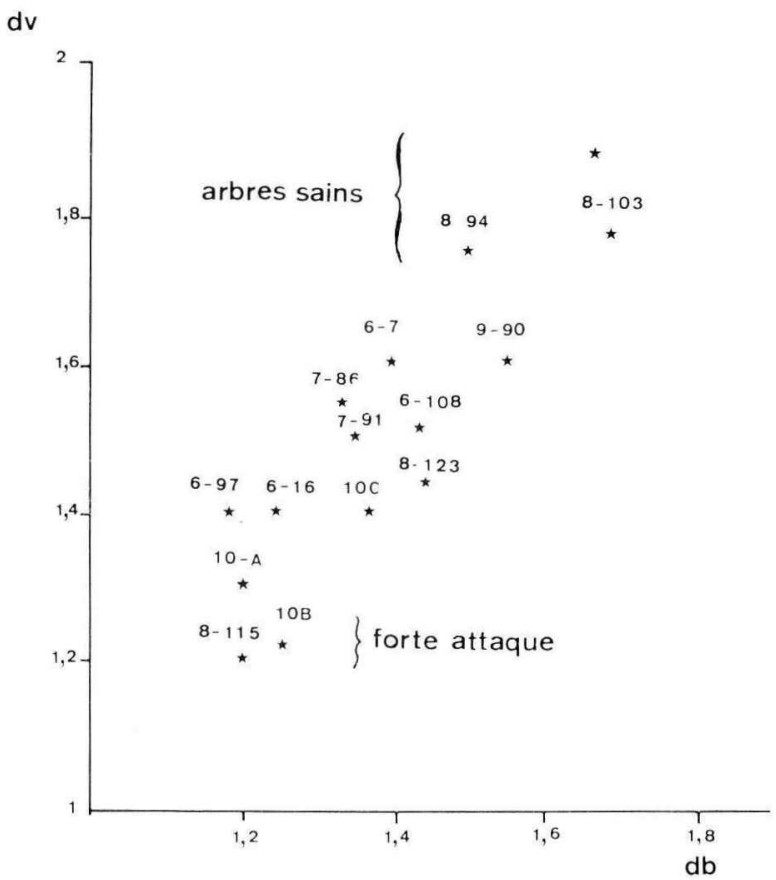

FIG. 7. - Classification de Pins maritimes en fonction des densités de deux couches ( $\mathrm{dv}$ et $\mathrm{db}$ ) du film IRC. Les arbres sains se différencient nettement des arbres très attaqués.

Classification of Maritime Pines according to optical densities of two layers ( $\mathrm{dv}$ and db) of the IRC film. Healthy trees are clearly differentiated from heavily attacked trees.

Un observateur au sol, à la condition que le peuplement ne soit pas trop fermé distingue relativement bien les arbres très dépérissants, par contre dans la catégorie des $d v \times d b$ compris entre 3,2 et 2,7 son classement est souvent mauvais, car il ne tient comple que des extrémités jaunissantes qui ne sont pas toujours le signe d'une population importante sur ce fût ; dans ce cas la méthode de télédétection donne des résultats plus sûrs.

Dans cette même classe des $d v \times d b$ compris entre 3,2 et 2,7 il est apparemment possible de distinguer les infestations moyennes (3 à 4 larves $L 2$ par $\mathrm{cm}^{2}$ de fût) des infestations fortes (5 à 10 larves L2 par $\mathrm{cm}^{2}$ ).

En finale on obtient sur les clichés le classement suivant :

$$
\begin{aligned}
& d v \times d b>3,2 \text { : arbres sains ou très peu infestés; } \\
& 3,2>\mathrm{dv} \times \mathrm{db}>3 \text { : arbres moyennement infestés ; } \\
& 3>\mathrm{dv} \times \mathrm{db}>2,7 \text { : arbres fortement infestés ; } \\
& \mathrm{dv} \times \mathrm{db}<2,7 \text { : arbres très dépérissants ; } \\
& \text { houppiers jaunes : arbres venant de mourir ; } \\
& \text { aiguilles tombées : arbres morts depuis plus de } 6 \text { mois. }
\end{aligned}
$$

La discrimination précoce de l'attaque et la distinction plus fine entre différents degrés d'attaque dépendent uniquement de la précision avec laquelle il est possible d'obtenir la variation de densité optique de chacune des couches. 
Les variations stationnelles prises en compte dans cette étude ne sont pas suffisantes pour fausser les résultats de l'analyse densitométrique : dans les 4 stations étudiées (Les Campaux, La Mole, Bagnols-en-Forêt, Saint-Cassien) les réponses des arbres sont identiques pour un même état sanitaire. Souvent la réponse spectrale des végétaux est partiellement modifiée en fonction des conditions pédologiques : si cette étude avait pris en compte des peuplements situés plus au Nord, dans la partie calcaire du massif, il est certain qu'il aurait fallu « recaler » l'étude densitométrique. La campagne C.N.E.S. de juin 1971 (†abl. 1) nous a montré que les Pins maritimes chlorosés avaient sur l'émulsion IRC une réponse très différente des pins sains (vol dans la région de Brignoles, Var).

II n'est pas conseillé d'étudier en densitométrie les arbres situés en bordures des clichés car ils ne sont pas vus sous le même angle que ceux situés au centre ef leurs caractéristiques densitométriques sont de ce fait faussée. Une prise de vue proche du midi-solaire ef l'utilisation d'un objectif de focale plus grande limitent cet inconvénient.

\section{5. - Essai de traitement automatique}

La classification des arbres en fonction du degré d'attaque par visualisation directe du film IRC et estimation des tonalités rouge-rosé est difficile même pour un observateur averti ; d'autre part d'un observateur à l'autre les classifications peuvent être différentes.

L'étude microdensitométrique des clichés en examinant successivement les houppiers d'un film est longue, et ne peut de ce fait être utilisée sur de grandes surfaces. De plus elle n'est possible que sur des peuplements clairs, dans lesquels les houppiers sont bien différenciés et de taille suffisante.

Un essai de classification automatique a été effectué avec l'aide de MM. Boissard et Valery du Laboratoire INRA de Télédétection de Versailles, sur un jeune peuplement très dense ayant des houppiers emmêlés; l'examen du cliché IRC ne permet pas le comptage du nombre d'arbres sains ef leur taille est trop réduite pour qu'ils soient étudiés en microdensitométrie (fig. $8 \mathrm{~A}$ ).

\section{1. - Technique}

Le film IRC subit une numérisation trichrome sur un microdensitomètre numérique à 256 niveaux avec un pas de $100 \mu$; une compression par 2 en lignes et colonnes aboutit à une résolution de $200 \mu$.

L'image numérisée est traitée sur une unité interactive Pericolor à microprocesseur en utilisant les critères de discrimination mis en évidence lors de l'étude microdensitométrique des clichés (cf. § 4.2).

Le sol nu recouvert d'aiguilles de pins desséchées a une réflectance voisine des arbres morts : l'ambiguïté a été levée en utilisant une combinaison des densités des couches verte et bleue différente de celle utilisée pour discriminer les degrés d'attaque par la cochenille. 

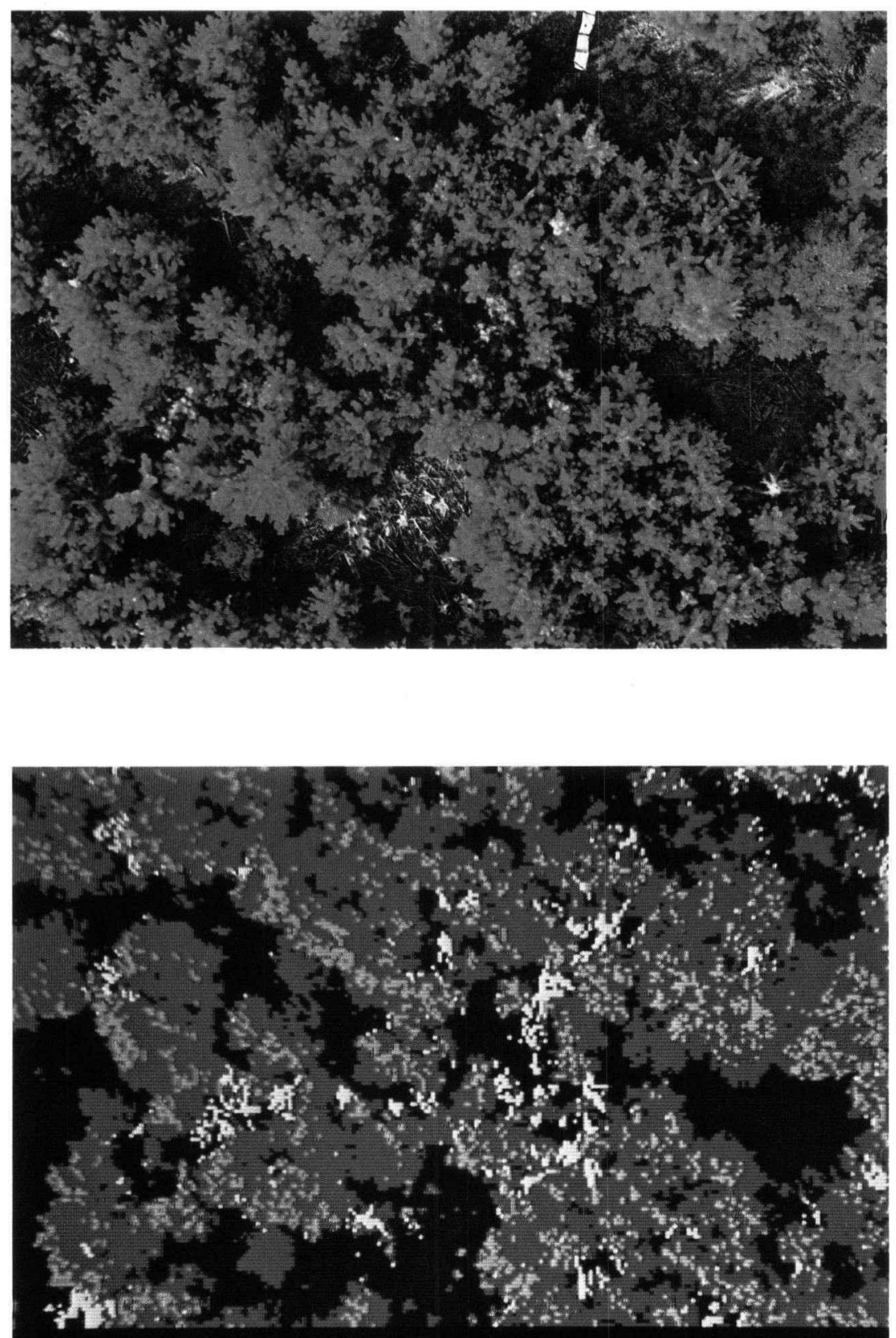

CLASSIFICATION D'APRES CLICHE IRC BAGNDLS 8- $09-71$ ARBRES SAINS

SDL

" ATtEINTS
" tREs ATtEINTS
" MDRTS




\section{2. - Résultats}

La figure $8 \mathrm{~B}$ montre le résultat d'une classification obtenue en combinant les composantes des trois couches, deux à deux, de façon itérative jusqu'à l'obtention de cinq classes finales. Le calcul de l'histogramme du rapport de deux canaux est visualisé sur écran et peut être facilement découpé en modes correspondant aux sous-classes créées.

Les cinq classes obtenues sont codées en couleur afin de faciliter la visualisation, la classe rose correspond aux arbres rose foncé sur le cliché IRC et la classe bleue aux arbres rose clair. Une fois la classification terminée la quantification du nombre de pixels appartenant à chacune des classes (tabl. 4) est automatique et le simple rapport végétation atteinte sur total de la végétation (dans ce cas 24,6 ) donne une bonne idée de l'intensité des attaques ; connaissant la taille moyenne des houppiers, exprimée en pixels la détermination du nombre d'arbres appartenant à chacune des classes devient facile.

\section{TABLEAU 4}

Résultat de la classification automatique, à partir du cliché IRC, comprenant 165 lignes de 256 pixels

Results of an automatic classification based on the IRC photograph with 165 lines of 256 pixels

\begin{tabular}{|c|c|c|c|}
\hline $\begin{array}{l}\text { Classe } n^{0} \\
\left(\text { Class } n^{0}\right)\end{array}$ & Caractéristiques (Characteristics) & $\begin{array}{c}\text { Nombre de } \\
\text { pixels } \\
\text { (Number of } \\
\text { pixels) }\end{array}$ & $\begin{array}{c}\text { Pourcentage } \\
\text { p. } 100\end{array}$ \\
\hline $\begin{array}{l}1 \\
2 \\
3 \\
4 \\
5\end{array}$ & $\begin{array}{l}\text { Arbres sains (Healthy trees) } \\
\text { Arbres atteints (Attacked trees) } \\
\text { Arbres très atteints (Heavily attacked trees) } \\
\text { Arbres morts (Dead trees) } \\
\text { Sol (Soil) }\end{array}$ & $\begin{array}{rr}21 & 856 \\
3 & 728 \\
2 & 058 \\
1 & 346 \\
13 & 274\end{array}$ & $\begin{array}{r}51,8 \\
8,6 \\
4,9 \\
3,2 \\
31,5\end{array}$ \\
\hline
\end{tabular}

Une fois la méthode de traitement mise au point, l'étude d'une nouvelle image d'une même scène est très rapide (quelques minutes) ; si la végétation spontanée varie

FIG. 8A. - Cliché IRC d'un peuplement de Pins maritimes attaqué par Matsucoccus feytaudi Duc. Les pins sains sont rouges; en fonction de leur degré d'attaque ils virent au rose, puis au rose clair; les arbres morts sont jaunes.

IRC photograph of a Maritime Pine stand attacked by Matsucoccus feytaudi Duc. Healthy pines are red ; according to the level of infestation, they turn pink to light pink; dead trees show in yellow.

FIG. 8B. - Le même peuplement après numérisation trichrome du cliché IRC en deux cent cinquante-six niveaux sur cent soixante-cinq lignes de deux cent cinquante-six pixels, traitement et codage coloré. Le nombre de pixels appartenant à chacune des classes est déterminé automatiquement (cf. tabl. 4).

The same stand after trichromatic digitisation of the IRC photograph into 256 levels on 165 lines of 256 pixels, processing and colored coding. The number of pixels in each class is determined automatically (see table 4). 
dans un même peuplement forestier, ou d'un peuplement à l'autre, il devient indispensable de superviser le traitement au début de chaque série de clichés pour vérifier qu'aucune confusion nouvelle due à la réflectance de la végétation spontanée n'est apparue.

\section{6. - Conclusion}

L'exploitation microdensitométrique trichrome des clichés IRC montre nettement que la réponse spectrale des Pins maritimes en cours d'attaque est différente de celle des arbres sains et qu'il est possible de discriminer plusieurs degrés d'attaque en utilisant les variations de densité optique de deux couches seulement de ce film. Un tel film à deux couches sensibles dans les deux bandes concernées a été réalisé à la suite de ces essais par Kodak. En URSS le film spectrozonal (désigné sous l'appellation SN-2 et récemment SN-6) a lui aussi deux couches ; l'une sensible dans le panchromatique, l'autre dans le proche infra-rouge. Dans un film à deux couches la richesse du rendu chromatique du diapofilm est moindre que dans un film à 3 couches, mais la comparaison de divers états sanitaires d'une même espèce végétale est meilleure (Olson, 1969 ; Kenneweg H., 1971).

Cette méthode permet :

- d'envisager une exploitation semi-automatique (en effet, il faut repérer individuellement chaque houppier) de clichés photographiques pour un inventaire de l'état sanitaire des arbres du peuplement ;

- de fournir des éléments d'information pour le choix des méthodes de traitement automatique.

Il était difficile, à l'époque où ce travail a été effectué, de disposer de scanners multispectraux. Le dépouillement des données de la campagne de 1972, l'une des premières du scanner dix canaux Daedalus en France, devrait permettre de progresser dans l'étude par télédétection des dégâts provoqués aux forêts par les ravageurs animaux et végétaux.

Le traitement automatique sur unité interactive à microprocesseurs, supprime le repérage individuel des houppiers indispensable en microdensitométrie trichrome, et grâce à la possibilité de quantification du nombre de pixels de chaque classe indique automatiquement pour chaque image la surface appartenant à chacune des classes repérées lors du traitement.

Certaines confusions, entre par exemple les arbres morts et les surfaces de sol éclairées et recouvertes d'aiguilles mortes, peuvent être éliminées par une adaptation des méthodes de traitement.

La méthode proposée pour cette étude de la détection des attaques de Matsucoccus feytaudi sur Pin maritime est extrapolable, à l'étude d'autres problèmes en forêt et en agriculture, en modifiant évidemment certains des critères de discrimination utilisés ici. Il faut cependant remarquer que l'altération de la réflectance spectrale n'est pas obligatoirement spécifique d'une attaque particulière (Heller, 1971; Riom, 1978) et que seul un contrôle au sol, et une bonne connaissance des sites d'étude, permet de déterminer la nature de l'attaque ; lorsqu'elle est connue la télédétection assure le suivi de cette attaque de façon répétitive, et sur de grandes surfaces.

Reçu pour publication en février 197.9. 


\title{
Remerciements
}

Ce travail a été effectué grâce au contrat DGRST, no 71.7.2624 « Reconnaissance multispectrale par survol d'attaques parasitaires en forêt »- «Remote sensing » (surveillance forestière) (responsable Ch. Goillot) pour la partie physique du programme, et au contrat, $n^{\circ} 68.01 .283$, avenant 3 (responsable J. Riom) pour la partie biologique ; ces deux contrats ont été accordés par le Comité « Lutte biologique $\gg$.

Nous remercions MM. Parent ef Perelgritz, de la Société Joyce Loebl France, pour leur assistance ainsi que $M$. Braud pour son travail technique effectuée sous la direction de $M$. Ch. Rossetti, que nous remercions tout particulièrement.

\section{Summary}

\author{
Remote sensing of Matsucoccus feytaudi Duc (Coccoidea, Margarodidae) \\ attacks in the Maritime Pine forests of southeastern France, \\ using trichromatic microdensitometry on IRC films
}

The level of Scale populations and the signs of attack were investigated on each individual tree of various Maritime Pine stands which had been selected for the development of a remote sensing investigation method using small scale $(1 / 250 ; 1 / 500 ; 1 / 2500)$, low altitude IRC photography $(37,75$ and $375 \mathrm{~m}$ ).

Tree crowns were analyzed by means of trichromatic microdensitometry ; the optical density of green $(\mathrm{dv})$ and blue $(\mathrm{db})$ layers evidences the degree of attack. Healthy trees have high $\mathrm{dv}$ and $\mathrm{db}$ values $(1,8$ and 2$)$; heavily infested trees have low $d v$ and $d b$ values $(1,2$ and 1,3$)$; trees undergoing an attack produce values between these two extremes. The red film layer (i. e. sensitive to Infra red) does not evidence the degree of attack. This method allows to distribute the trees through six classes.

The IRC digitized film with 256 levels using trichromatic densitometry is processed through an interactive unit based on micro-processing. This allows an automatic classification and quantifying of pixels corresponding to various degrees of the attack in the stand.

\section{Références bibliographiques}

BENZON M. L. et SIMS W. G., 1967. False-color film fails in practice. J. of Forestry, 65, 904.

CIESLA W. M., BELL J. C. et CURLIN J. W., 1967. Color photos and the southern pine beetle. Photogr. Engr., 31, 883-888.

DOVERSPIKE G. E., FLYNN E. M. et HELLER R. C., 1965. Microdensitometer applied to land use classlfication. Photogr. Engr., 31, 2, 294-306.

GOILLOT Ch., ROSSETTI Ch., RIOM J. et FABRE J. P., 1977. Evaluation par microdensitométrie trichrome de clichés IRC pour la télédétection d'attaques de cochenilles (Matsucoccus feytaudi Duc.) dans les forêts de Pin maritime du Sud-Est de la France. C. R. des journées de Télédétection, Toulouse, 26-28 oct. 1976, 81-98.

HANSEN J. H., 1973. Techniques for determining color differences of imaged vegetation on color IR-film. Symposium IUFRO, S.6-05, Freiburg.

HELLER R. C. et BEAN J. L., 1952. Aerian surveying methods for detecting forest insect ourbreaks. V.S.A. Depart. Agr. BEPQ Div. of Forest Insects (mileo).

HELLER R. C., 1971. Detection characterization of stress symptoms in forest vegetation. Proc. intern. Workshop on earth resources survey systems. Ann. Arbor. mai 1971, Vol. II, 109-150.

HELLER R. C., 1971. Color and false-color photography : its growing use in forestry. Application of remote sensors in forestry. IUFRO, S.05, XVe Congrès, 57-73.

KNIPLING E. B., 1968. Leaf reflectance and image formation on color infra-red film. Annual meeting, Ecol. Soc. of Amer., Madison, Wis. 1968.

KENNEWEG H., 1971. Color and false-color photography ; its growing use in forestry. Application of remote sensors in forestry. IUFRO, S. 05, XVe Congrès, 57-73.

MEYER M. P. et FRENCH D. W., 1967. Detection of diseased trees. Photogr. Engr., 33, 1035-1040.

MURTHA P. A. et HAMILTON L. S., 1969. Defection of simulated damage on conifers using near infra-red film. Journal of forestry, 827-829. 
MURTHA P. A., 1976. Inventory and monitoring of forest diseases and damages by remote sensingconsiderations about promising ways to do it. Remote sensing in Forestry XVI, IUFRO Congress, Oslo, 21-26 juin 1976.

RIOM J., FABRE J. P., BOULBRIA A. et GERBINOT B., 1971. Défection d'attaques parasitaires en forêts de Pins maritimes dans les Maures, l'Estérel et les Landes. Colloque du Centre National d’Efudes Spatiales, Paris, 26-27 oct. 1971 «La Télédétection des Ressources Naturelles».

RIOM J. ef GERBINOT B., 1977. Etude biologique et écologique de la cochenille du Pin maritime Matsucoccus feytaudi Duc., 1942 (Coccoidea, Margarodidae, Xylococcinae) dans le sud-est de la France. I. - Biologie Générale et Phénologie. Ann. Zool. Ecolo. Ani., 9 (1), 11-50.

RIOM J., 1978. Aperçu des méthodes de la télédétection phytosanitaire. Bulletin de la Saciété Française de Photogramétrie, $\mathrm{n}^{0}$ 61, 21 p.

RIOM J., 1977. Etude biologique et écologique de la cochen ille du Pin maritime Matsucoccus feyłaudi Duc., 1942 (Coccoidea, Margarodidae, Xylococcinae) dans le sud-est de la France. III. Structure des populations sur l'arbre, Ann. de Zool. Ecolo. Ani., 9 (4), 30 p. (sous presse).

RODRIGEZ-BEJARANO D., 1975. Density slicing applied to Forest type delineation. Photogr. Engr. and Remote sensing, 41, 8, 1029-1037.

ROSSETTI, KOWALISKI, HAVE, 1966. Relations entre les caractéristiques de réflexion spectrale de quelques espèces végétales et leurs images sur les photographies aériennes en couleur, terrestres et aériennes. Symp. Infer. de Photointerprétation II, 25-50.

ROSSETTI Ch., 1967. Compte rendu de mission sur l'étude des images photographiques aériennes à grande échelle de diverses formations végétales. CNRS-CEPE, $120 \mathrm{p}$.

TARKINGTON R. G., SOREM AI., 1963. Color and false-color film dor aerial photography. Photogr. Engr., 29, 88-95. 